\title{
An inexpensive, customizable microscopy system for the automated quantification and characterization of multiple adherent cell types
}

Vishwaratn Asthana ${ }^{\text {Corresp., }}{ }^{1}$ ， Yuqi Tang ${ }^{1}$, Adam Ferguson ${ }^{1}$, Pallavi Bugga ${ }^{1}$, Anantratn Asthana ${ }^{2}$, Emily R

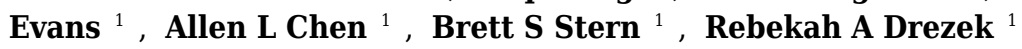

1 Department of Bioengineering, Rice University, Houston, Texas, United States

2 Department of Molecular \& Cell Biology, University of California, Berkeley, Berkeley, California, United States

Corresponding Author: Vishwaratn Asthana

Email address: vishwaratn.asthana@rice.edu

Cell quantification assays are essential components of most biological and clinical labs. However, many currently available quantification assays, including flow cytometry and commercial cell counting systems, suffer from unique drawbacks that limit their overall efficacy. In order to address the shortcomings of traditional quantification assays, we have designed a robust, low-cost, automated optical cell cytometer that quantifies individual cells in a multiwell plate using tools readily available in most labs. Plating and subsequent quantification of various dilution series using the automated microscopy-based cytometer demonstrates the single-cell sensitivity, near-perfect $\mathrm{R}^{2}$ accuracy, and greater than 5-log dynamic range of our system. Further, the microscopy-based cytometer is capable of obtaining absolute counts of multiple cell types in one well as part of a co-culture setup. To demonstrate this ability, we recreated an experiment that assesses the tumoricidal properties of primed macrophages on co-cultured tumor cells as a proof-of-principle test. The results of the experiment reveal that primed macrophages display enhanced cytotoxicity towards tumor cells while simultaneously losing the ability to proliferate, an example of a dynamic interplay between two cell populations that our microscopy-based cytometer is successfully able to elucidate. 
1 An Inexpensive, Customizable Microscopy System for the Automated Quantification and

2

3

4 Vishwaratn Asthana $^{1 *}$, Yuqi Tang ${ }^{1 \uparrow}$, Adam Ferguson ${ }^{1 \uparrow}$, Pallavi Bugga ${ }^{1}$, Anantratn Asthana ${ }^{2}$,

5 Emily R. Evans ${ }^{1}$, Allen L. Chen ${ }^{1}$, Brett S. Stern ${ }^{1}$, Rebekah A. Drezek ${ }^{1}$

6

$7{ }^{1}$ Department of Bioengineering, Rice University, Houston, Texas, United States of America, ${ }^{2}$

8 Berkeley University, Berkeley, California, United States of America

$9 *$ Corresponding author

10 E-mail: vishwaratn.asthana@,rice.edu (VA)

11 T These authors contributed equally to this work.

12

13

14

15

16

17

18

19

20

21

22 
23 Cell quantification assays are essential components of most biological and clinical labs.

24 However, many currently available quantification assays, including flow cytometry and

25 commercial cell counting systems, suffer from unique drawbacks that limit their overall efficacy.

26 In order to address the shortcomings of traditional quantification assays, we have designed a

27 robust, low-cost, automated optical cell cytometer that quantifies individual cells in a multiwell

28 plate using tools readily available in most labs. Plating and subsequent quantification of various

29 dilution series using the automated microscopy-based cytometer demonstrates the single-cell

30 sensitivity, near-perfect $\mathrm{R}^{2}$ accuracy, and greater than 5-log dynamic range of our system.

31 Further, the microscopy-based cytometer is capable of obtaining absolute counts of multiple cell

32 types in one well as part of a co-culture setup. To demonstrate this ability, we recreated an

33 experiment that assesses the tumoricidal properties of primed macrophages on co-cultured tumor

34 cells as a proof-of-principle test. The results of the experiment reveal that primed macrophages

35 display enhanced cytotoxicity towards tumor cells while simultaneously losing the ability to

36 proliferate, an example of a dynamic interplay between two cell populations that our

37 microscopy-based cytometer is successfully able to elucidate.

38

39

40

41

42

43

44

45 

67 2013).

\section{Introduction}

Cell quantification assays are essential components of most biological labs, and are used for a variety of applications, including cytotoxicity, viability, and proliferative studies. Though these assays have improved significantly since the advent of hemocytometers, they still suffer from a number of apparent limitations. Current cell quantification assays can be divided into two major classes: metabolic and cell counting. Metabolic assays, though originally designed to assess cell viability, are often used to indirectly assess cell number. Metabolic assays like MTT (3-(4,5-dimethylthiazol-2-yl)-2,5-diphenyltetrazolium bromide) or alamarBlue, both of which use the reductive environment of the cell to convert dye reagents into detectable colored products, are relatively easy to perform, and can provide additional information on cell health that a counting assay may not. However, they are not ideal for certain experimental setups, as they have a limited dynamic range and are prone to confounding interference in the presence of certain chemicals (Chakrabarti et al. 2000; Doak et al. 2009; Hamid et al. 2004; Ulukaya et al. 2004; Vistica et al. 1991). These assays also do not always align well with the DNA content of the cell —a parameter that correlates strongly with cell number-limiting the cell quantification potential of these assays (Quent et al. 2010). Cell counting assays on the other hand, though generally more manually intensive, are more representative of actual cell counts than metabolicbased proxy assays (Chan et al. 2013). Flow cytometry, often considered the gold standard for cell counting and analysis, is an especially powerful technique for quantifying individual cells, and is one of the few modalities capable of identifying cell population counts in both a monoculture and co-culture setup (Gedye et al. 2014; Gerashchenko 2008; Gerashchenko \& Howell 

areas of research could benefit immensely from co-culture setups-including biomaterials, immunology, and cancer biology — if better characterization and quantification methods were available for these studies (Bidarra et al. 2011; Miki et al. 2012). Most approaches to co-culture are restrictive, and often require that plated cells be physically separated via a transwell insert or microfluidic chamber that only permits the exchange of media (Arrigoni et al. 2016; Goers et al. 2014; Katt et al. 2016). Yet physical contact has been shown to be important for studying the interactions of many cell types in a variety of physiological contexts (Cruickshank et al. 2004; de Goer de Herve et al. 2010; Gerashchenko \& Howell 2003; Holt et al. 2010; Suzuki et al. 2004). Most cell populations do not behave independently and a better understanding of the interaction between multiple cell types in a system will help improve our understanding of many physiological phenomena.

Most studies that utilize a true co-culture setup with physical contact rely on flow cytometry to quantify individual cell types. Unfortunately, flow cytometry has several drawbacks that apply not only to co-culture setups but mono-culture setups as well. As a starting point, flow cytometers are fairly sophisticated; as a result, these instruments are generally expensive and often require skilled upkeep (Nasi et al. 2015). Flow cytometry also requires cells to be in suspension - thus the majority of experiments that are conducted on adherent cells in multiwell plates require trypsin treatment for cell detachment. Trypsin, however, can damage cells and

87 cleave extracellular markers that may be used for cellular identification or other forms of 88 analysis (Gedye et al. 2014). Additionally, certain cell types are not amenable to trypsin 89 treatment and as a result require manual cell scraping, a process that is prone to human error. 90 Cell scraping can also mechanically damage some cell types, leading to erroneous results with 
91 certain assays that are used in combination with flow cytometry: for example, false positives

92 with membrane permeable cell viability assays such as propidium iodide (Batista et al. 2010;

93 Bundscherer et al. 2013). Furthermore, cells in suspension have the potential to stick to each

94 other forming aggregates that can interfere with analysis. All together, the need to bring adherent

95 cells into suspension makes flow cytometry less than ideal for many types of studies.

96 An additional limitation specific to co-culture setups is that typical flow cytometry

97 obtains relative counts rather than absolute counts, i.e. each cell type is expressed as a percent of

98 the total sample assayed. This may generate misleading results when comparing counts for

99 multiple cell types between conditions.

To address the shortcomings of the various cell quantification assays, we have put

101

102

103

104

105

106

109

110

111

112 Spink et al. 2006). 
It should be noted that other optical counting systems have been developed to address the

114 aforementioned limitations of flow cytometry. However, the optical system we present here still

115 bears a number of advantages over these pre-sold cytometric platforms. First, for labs that

116 already possess a fluorescent microscope, the microscopy-based cytometer is a relatively small

117 investment to the overhead cost of buying a prebuilt optical counting system or flow cytometer.

118 Further, unlike most commercial counting systems, the microscopy-based cytometer discussed

119 here is flexible in the assays and cell types that can potentially be analyzed, and highly

120 customizable in both setup and analysis, permitting the extraction of more relevant and robust

121 information per experiment. In this way, biological workflow is not limited by the vendor-

122 specific restrictions of pre-built systems, but rather expanded to include all functionalities of

123 standard fluorescent microscopes.

124 Many image cytometers currently on the market also exhibit a smaller dynamic range and

125 reduced sensitivity compared to the system we present here. Lastly, most commercial cytometric

126 platforms are not able to discriminate multiple distinct cell populations, with potentially complex

127 morphologies, within a single well. This is largely due to the inability of most commercial

128 cytometers to accurately segment non-spherical morphologies, thus restricting their applicability

129 for most co-culture setups. A more in depth comparison of our microscopy-based cytometer to

130 commercially available systems, including in cost, can be found in the discussion as well as

131 supplementary section (Text S1).

132 Materials and Methods

133 Cell Culture and Plating

134 JC CRL 2116 mouse adenocarcinoma cells were obtained from ATCC (American Type

135 Culture Collection; Manassas, Virginia) and maintained in Dulbecco's Modified Eagle Medium 
136 (DMEM) supplemented with 10\% Fetal Bovine Serum (FBS) and 1\% penicillin/streptomycin.

137 J774.A1 mouse macrophages were obtained from ATCC and maintained in Roswell Park

138 Memorial Institute Medium (RPMI), supplemented with 10\% Fetal Bovine Serum (FBS) and 1\%

139 penicillin/streptomycin. Both cells were grown at $37^{\circ} \mathrm{C}$ in $5 \% \mathrm{CO}_{2}$.

140 For plating, cells were trypsinized (JC CRL 2216 cells) or scraped (J774.A1 cells) from

141 their flasks and quantified manually using a bright-line hemocytometer (Sigma-Aldrich; St.

142 Louis, Missouri). Due to the variability present in counts obtained using a hemocytometer,

143 established values were primarily used to determine the approximate concentration of the

144 primary stock from which precise subsequent dilutions were performed. Dilution series were

145 generated by pulling cells from the previous stock and diluting in fresh media. Triplicates of each

146 dilution were then plated on either a 48- or 12-well Corning Costar flat bottom cell culture plate

147 (Thermo Fisher Scientific; Waltham, Massachusetts) and given 24 hours to attach to the plate

148 surface.

\section{Co-Culture Experiments}

$150 \quad$ J774.A1 cells were seeded at $2.5 \times 10^{4}$ cells/well in RPMI media in a 48-well plate.

151 Immediately following seeding, cells were exposed either to $1 \mu \mathrm{g} / \mathrm{mL}$ of lipopolysaccharide

152 (LPS) (Sigma-Aldrich; St. Louis, Missouri), $0.1 \mu \mathrm{g} / \mathrm{mL}$ of mouse interferon gamma (IFN $\gamma$ )

153 (BioLegend; San Diego, California), both $1 \mu \mathrm{g} / \mathrm{mL}$ of LPS and $0.1 \mu \mathrm{g} / \mathrm{mL}$ of IFN $\gamma$, or neither.

154 After a 24-hour incubation, the wells were washed twice with Phosphate Buffered Saline (PBS).

155 JC CRL 2116 cells were then added at $1 \times 10^{4}$ cells/well in RPMI media to every well. Cells were 156 processed and imaged after 24 hours.

157 Cell Staining 
159 prior to imaging. After firm cell adhesion, media was removed from the wells by inverting the 160 plate and cells were fixed for 15 minutes in BD Cytofix (BD Biosciences; San Jose, California).

161 Fixative was removed by washing the plate twice with Hank's Balanced Salt Solution (HBSS).

162 Cells were then stained for 5 minutes in a $2.5 \mu \mathrm{g} / \mathrm{mL}$ DAPI stain solution (Thermo Fisher

163 Scientific; Waltham, Massachusetts). The plate was again washed twice with HBSS and finally 164 resuspended in HBSS for imaging.

165 Experiments involving multiple stains, including nuclear, cytoplasmic, and

166 surface/antibody stains, required a slightly different protocol. Using the immune co-culture

167 experiment as an example, JC CRL 2116 cells were stained in their cell culture flask with $20 \mu \mathrm{M}$

168 of Vybrant CFDA SE (Invitrogen; Carlsbad, California) the day prior to plating using the

169 recommended protocol. After completion of the experiment and immediately prior to imaging,

170 media was removed from the wells by inverting the plate, and the plate was subsequently

171 blocked with a 1\% bovine serum albumin (BSA) (Sigma-Aldrich; St. Louis, Missouri) solution

172 in HBSS for 15 minutes. HBSS supplemented with calcium was found to help prevent cell

173 detachment prior to cell fixation. Cells were then incubated with a $5 \mu \mathrm{g} / \mathrm{mL}$ PE anti-mouse

174 CD11b antibody (BioLegend; San Diego, California) diluted in 1\% BSA HBSS for 1 hour to

175 stain the J774.A1 cells. After incubation, the antibody solution was removed by inverting the

176 plate after which the wells were washed twice with $1 \%$ BSA HBSS. The cells were then fixed for

17720 minutes in BD Cytofix. Fixative was removed by washing the plate twice with HBSS. Cells

178 were then stained for 5 minutes in a $2.5 \mu \mathrm{g} / \mathrm{mL}$ DAPI stain solution. The plate was again washed

179 twice with HBSS and finally resuspended in HBSS for imaging.

180 Acquiring Whole-Well Images 
182 with motorized x, y, and z stage (Nikon Instruments Inc.; Melville, New York). Images were

183 captured using a NAMC 10x objective and Andor Zyla 4.2 sCMOS camera (Andor Technology;

184 Belfast, Northern Ireland). DAPI/Hoechst (excitation: 360/40, emission: 460/50, dichroic mirror:

185 400), GFP (Ex: 470/40, Em: 525/50, DM: 495), and Texas Red (Ex: 560/55, Em: 645/75, DM:

186 595) filter cubes (Nikon Instruments Inc.; Melville, New York) were used to image cells stained

187 with DAPI, Vybrant CFDA SE, and PE anti-mouse CD11b antibody respectively. Using the

188 associated Nikon software, NIS-Elements, an automated macro was set up for whole-well

189 acquisition. First, an x-y coordinate list demarcating the center of every well was generated by

190 manually determining the center of the first and last well of the plate and dividing these values

191 by the number of rows and columns. The coordinate list will vary based on the type of multiwell

192 plate but only needs to be generated once. Using a $10 \times$ objective and 1,600×1,600 pixel region of

193 interest $(\mathrm{ROI}), 10 \times 10$ images for a 48 -well plate and $19 \times 19$ images for a 12 -well plate were tiled

194 together to create a whole-well image. The center of each well serves as the origin point of the

195 tiled images as well as the autofocus point.

\section{Processing Whole-Well Images}

197 Images were preprocessed using ImageJ (National Institute of Health; Bethesda,

198 Maryland). The ring of autofluorescence around the well edges was removed using the Subtract

199 Background function with a rolling ball radius of 50 pixels. Fluorescent channels that were too

200 faint for analysis were occasionally made brighter using the Enhance Contrast function. If

201 images were stitched during image acquisition they were subsequently cropped into 100 smaller

202 images using the Montage to Stack function at which point they were transferred to CellProfiler

203 (Broad Institute; Cambridge, Massachusetts), specifically CellProfiler version 2.2.0, for 
204 segmentation. ImageJ macros (Macro S1) and CellProfiler codes (CellProfiler Code S1, and

205 CellProfiler Code S2) are provided as supplementary files. A more detailed explanation of

206 relevant ImageJ and CellProfiler functions can be found in the supplementary section (Text S1).

207 Statistics

208 All experiments were conducted in triplicates. Error bars represent the standard deviation

209 of replicate experiments. Linear regressions and associated coefficients of determination $\left(\mathrm{R}^{2}\right)$

210 were derived using Excel. For the JC CRL 2116 and J774.A1 co-culture experiment, Turkey’s

211 HSD test (JMP Pro 13) was used to calculate statistical significance (set at $\alpha=0.05$ ) between

212 conditions.

\section{Results}

214 Accuracy of Nuclear Quantification in Conjunction with Whole-Well Imaging

Many studies take representative or random images that represent only a small portion of

216 the entire well to make a claim about differences in cell count/viability between conditions.

217 Instead of manually acquiring a series of random or representative images, which is both tedious

218 and potentially inaccurate, it is possible - using a fluorescent microscope with a motorized $\mathrm{x}, \mathrm{y}$,

219 and $\mathrm{z}$ stage - to automate capture of the whole well using movement in the $\mathrm{x}$ - and y-plane to

220 image the well and movement in the z-plane to autofocus (Fig 2).

221 To count individual cells, wells were stained with a DAPI nuclear stain. Nuclei are often

222 roundly shaped and spaced from adjacent nuclei by the cell cytoplasm and membrane, making

223 segmentation relatively straightforward. In addition, there is usually one nucleus per cell, making

224 nuclear segmentation ideal for cell counting. To demonstrate the power of nuclear counting and

225 whole-well imaging, a linear dilution series of JC CRL 2116 mouse adenocarcinoma cells going

226 from 10,000 cells/well all the way down to 1,000 cells/well was plated; an experiment spanning 
227 one order of magnitude. Three linear curves were then generated from five random images, a box

228 crop of the whole-well image, or the whole-well image itself, and used to determine cell count

229 (Fig 3). Accuracy improves significantly as the percentage of the experimental space being

230 assayed increases, with whole-well imaging displaying a near-perfect $\mathrm{R}^{2}$ and minimal error

231 between replicates.

\section{Establishing Dynamic Range and Sensitivity}

The optical cell cytometer obtains accurate counts by counting individual cells. In theory

234 it should be able to count anywhere from one cell to the confluency limit of the well plate the

235 experiment is conducted in with single-cell precision. To demonstrate the dynamic range of the

236 system, a dilution series of JC CRL 2116 cells going from 100,000 cells/well all the way down to

237100 cells/well were plated in a 12-well plate; an experiment spanning three orders of magnitude

238 (Fig 4). The system performs strongly within the confluency limit of the plate with a dynamic

239 range limited only by the surface area of the multiwell.

240 To validate the sensitivity of the system, a linear dilution series starting at 100 cells/well

241 and going down to 1 cell/well was also plated. However it was found that at such low

242 concentrations, it was not feasible to reliably plate the desired number of cells. Accordingly, the

243 cells that were plated were manually counted in the brightfield channel and compared with

244 CellProfiler counts obtained from segmenting nuclei in the DAPI channel (Fig 5). The system

245 demonstrates single-cell sensitivity and resolution with most deviations attributable to human

246 error when manually counting.

\section{Counting Using Surface and Cytoplasmic Stains}

248 In order to count multiple cell types in a co-culture experiment, additional cell stains are

249 required. Cell stains can be grouped into one of three categories: nuclear, surface, and 
250 cytoplasmic. Because most nuclear stains non-specifically stain DNA and compromise cell

251 viability, they cannot be used to differentiate cell types. Instead, vital cytoplasmic dyes like

252 Vybrant CFDA SE (Vybrant carboxyfluorescein diacetate succinimidyl ester), or antigen-

253 specific surface stains, such as fluorophore-conjugated antibodies, need to be used.

254 Surface and cytoplasmic staining alone can be used to differentiate cells, but primary

255 segmentation of these stains is fairly difficult. Factors including inhomogeneous staining, cell

256 contact with neighboring objects, and complex cell morphologies make segmentation less than

257 ideal. One way to overcome this limitation is to use easily identifiable and spatially resolvable

258 nuclei delineated in one fluorescent channel as a seed/primary object to guide detection of the

259 cell border/secondary object outlined in a separate fluorescent channel (Jones et al. 2005;

260 Vincent \& Soille 1991). However, when plating more than one cell type, for example in a co-

261 culture setup, this approach alone is insufficient because every nuclei will generate a secondary

262 object regardless of whether there is an associated cell in CellProfiler (Carpenter et al. 2006). To

263 overcome this limitation, several additional layers of image processing, including mask

264 generation, need to be performed; these are elaborated upon in the discussion section.

265 To validate the power of this approach as well as the efficacy of cytoplasmic and surface

266 staining, a linear dilution series of J774.A1 cells, a mouse macrophage cell line, was plated going

267 from 10,000 cells/well down to 1,000 cells/well. These cells were stained with Vybrant CFDA

268 SE (cytoplasmic stain) prior to plating, and phycoerythrin (PE)-conjugated anti-CD11b

269 antibodies (surface stain) as well as DAPI (nuclear stain) immediately prior to imaging. The

270 system performs less than ideally when segmenting the cytoplasmic or surface stains alone (Fig

271 S1). When nuclei are used as seeds however, the microscopy-based cytometer performs robustly

272 both for cytoplasmic and surface staining (Fig 6). 


\section{Quantification of Multiple Adherent Cell Types}

274 To demonstrate the ability of the optical cell cytometer to determine absolute counts of

275 multiple cell types in a single well, we recreated a macrophage-tumor co-culture immunology

276 experiment that was conducted in 1991 by Novotney, et al. as a proof-of-principle test. JC CRL

2772116 tumor cells were labeled with Vybrant CFDA SE in their cell culture flask 24 hours prior to

278 plating. J774.A1 macrophages were plated first and were either left unprimed or primed with

279 either LPS, interferon gamma (IFN $\gamma$ ) — a known macrophage activator, or both (Schoenborn \&

280 Wilson 2007). After a 24-hour incubation, LPS and IFN $\gamma$ were removed from the wells and the

281 previously stained JC CRL 2216 cells were added. After another 24-hour incubation, the wells

282 were stained with a PE-conjugated anti-CD11b targeting antibody to specifically label the

283 J774.A1 macrophages, as well as DAPI to stain all nuclei.

284

The results of the test reveal an interesting relationship between macrophage activation

285 and tumoricidal activity. The more strongly primed the macrophages (LPS+IFN $\gamma$ versus

286 unprimed), the more pronounced the tumoricidal response $(P<0.05)$; however, strongly primed

287 macrophages also lose the ability to proliferate, indicated by a sharp drop in macrophage count

$288(P<0.05)($ Fig 7a). Absolute counts of both cell populations show that erroneous results would

289 have been obtained by any setup that looks at relative counts (Fig 7b). Cytometric analysis

290 reveals that primed J774.A1 macrophages are also larger in size $(P<0.05)($ Fig $7 \mathrm{c})$, and appear

291 to have either increased uptake or phagocytic activity, as demonstrated by the retention of

292 Vybrant dye that was initially present in the cytoplasm of the JC CRL 2116 tumor cells $(P<$

293 0.05) (Fig 7d). CD1 1b expression levels do not appear to increase under these experimental

294 conditions $(P>0.05)$ (Biswas \& Sodhi 2002).

\section{Discussion}


We present here a relatively simple optical counting setup that brings together several

297 established techniques — including automated microscopy, cellular staining, and cell

298 segmentation - to quantify every cell in an experimental space, without the need for

299 trypsinization or cell scraping. Using whole-well imaging, we demonstrate that taking

300 representative or random images of a well to make a claim about cell viability/counts may be

301 misleading given the non-uniform distribution of cells in a well. In addition, by counting each

302 individual cell using nuclear segmentation, we demonstrate the impressive accuracy and

303 resolution of our optical quantification system. In general, counting single events/cells provides a

304 resolution that cannot be obtained with bulk ensemble measurements, and can significantly

305 improve sensitivity (Chang et al. 2012; Rissin et al. 2010). This approach, in conjunction with

306 whole-well imaging, also offers increased statistical power as the experimentally relevant region

307 is sampled in its entirety.

308 Next, we show that the system can accurately quantify cells within the confluency limit

309 of the plate. By increasing the surface area of each well however, it is possible to expand the

310 working range of the microscopy-based cytometer. Though 12 -well plates were used here to

311 accurately quantify up to 100,000 cells/well, it is possible to use 6-well plates or less, with a

312 larger corresponding surface area per well, to push the upper limits of detection.

313 On the lower end of the microscopy-based cytometer's operating range, illumination

314 correction, and sufficient post-stain washing make counting of just a single-cell theoretically

315 possible with minimal interference from background or noise. Data from tests that were

316 conducted with only a few cells per well demonstrate the single-cell sensitivity of the system, but

317 slight deviations in segmented nuclei-derived counts versus manual counts are evident as

318 demonstrated by the imperfect $\mathrm{R}^{2}$ and non-unity slope. While manual counts were performed 
319 solely on brightfield images, merging of nuclei in the DAPI channel with cell outlines in the

320 brightfield channel reveal that deviations in the lower range data were actually due to human

321 error. Ultimately, counts obtained via CellProfiler proved more reliable than manual counting

322 validating the ability of the microscopy-based cytometer to quantify down to the single-cell

323 level. Systems with improved sensitivity are not only advantageous when conducting screening

324 assays on smaller sized plates (such as 96-, 384-, and 1536-well plates), but also when detecting

325 a very low concentration of cells on large well plates. A sensitive system may also prove useful

326 for detecting rare cell subpopulations in a heterogeneous group (Lin et al. 2011).

327 Testing of the upper and lower extremes of the microscopy-based cytometer reveal the

328 greater than 5-log detection range that can be achieved with the instrument. Systems with a large

329 dynamic range are particularly advantageous for screening cytotoxic compounds such as

330 antineoplastic agents. It has been shown that compounds that reduce cell viability by at least two

331 orders of magnitude in vitro are more likely to demonstrate a response in clinical trials, making

332 an assay with an even larger dynamic range more appealing (Frgala et al. 2007). All together, the

333 combination of improved sensitivity, resolution, and dynamic range afforded by the automated

334 microscopy-based cytometer opens up the possibility of a new set of cell culture experiments that

335 were not previously feasible.

336 We next show that certain cytoplasmic and surface stains, in conjunction with a nuclear

337 stain, can be used to effectively differentiate cell populations in a co-culture setup. Vybrant

338 CFDA SE dyes, for example, are designed to form intracellular fluorescent conjugates that

339 homogenously stain the cell cytoplasm, are well-retained, and are not transferred to adjacent

340 cells or passed onto daughter cells during division (Bronner-Fraser 1985; Hodgkin et al. 1996;

341 Lyons \& Parish 1994; Nose \& Takeichi 1986; Weston \& Parish 1990). Alternatively, cells can 
342 be stained post-experiment and just prior to imaging using target-specific dyes, such as

343 fluorescently-conjugated antibodies.

344 For counting of more than one cell type in a co-culture setup, using a mask generated

345 from the fluorescent outlines of either a surface or cytoplasmic stain to delineate which nuclei

346 belong to which cell type proved to be the most robust. To do this, whole-cell fluorescence-

347 generated from either a surface or cytoplasmic stain - is used to create an inclusive mask that

348 retains nuclei contained within. This method can be repeated iteratively for each cell specific

349 stain, eventually grouping every nucleus with its associated cell population. These filtered nuclei

350 images can then be segmented and quantified using standard nuclear segmentation to generate

351 respective counts for each cell type. This approach is made possible using the fluorescent

352 staining and algorithmic combination proposed herein, and overcomes many of the limitations of

353 traditional segmenting systems. A sample workflow of this process with associated images can

354 be found in the supplementary section (Fig S2).

355 It should be mentioned that many commercial image cytometers largely rely on primary

356 segmentation for the quantification of multiple adherent cell types in a single well (Pozarowski et

357 al. 2013). This often requires the utilization of an array of algorithmic functions such as

358 thresholding, contouring, water shedding, cleaning, eroding, dilating, opening, closing, and

359 smoothing to uniquely identify individual cells. Classifiers (both object and pixel) are also

360 occasionally utilized to train systems to recognize user-delineated objects. However, these

361 algorithmic approaches are largely inadequate for quantifying cells with complex morphologies

362 ultimately limiting the accuracy and performance of commercial systems in these settings. For

363 our study in particular, primary segmentation of the cytoplasmic and surface stains alone

364 performed reasonably well when cell morphology was round and staining was homogenous (as 
365 was the case with the J774.A1 triple stain). When cell morphology became more spindly (as seen

366 with JC CRL 2116 cells), or staining became more inhomogeneous (as seen with activated

367 J774.A1 cells) however, a marked decrease in primary segmentation performance was observed

368 (data not shown) indicating that the aforementioned algorithmic functions are limited in their

369 generalizability. Using the fluorescent outline of the cell to generate a mask removes the need for

370 primary segmentation of these stains, and instead harnesses the accuracy/power of nuclear

371 segmentation, allowing for highly accurate quantification of multiple cell types with varying

372 morphologies in a co-culture setup. If a user was already in possession of a commercial optical

373 counting system, the proposed algorithmic approach could be adapted to achieve the same

374 results.

375 The ability of the microscopy-based cytometer to accurately count individual cells of a

376 specific population is best demonstrated by the macrophage-tumor co-culture experiment we

377 recreated. In 1991, Novotney, et al. set out to determine the tumoricidal properties of J774.A1

378 mouse macrophages when primed with lipopolysaccharide (LPS). To do this, the target tumor

379 cell line needed to be radiolabeled with ${ }^{51} \mathrm{Cr}$ then co-cultured with primed J774.A1 macrophages.

380 The extent of tumor killing was determined by measuring the increase in radioactivity of the

381 supernatant due to tumor cell death and detachment (Novotney et al. 1991). Unfortunately, the

382 use of ${ }^{51} \mathrm{Cr}$ to radiolabel target tumor cells is expensive and manually intensive by today's

383 standards. While cell-permeable fluorogenic protease substrates have been developed as a

384 replacement for ${ }^{51} \mathrm{Cr}$, neither is capable of dynamically quantifying changes in effector cell

385 (macrophage) count and the tumor cells they target (Packard \& Komoriya 2008). A suitable

386 alternative assay to count both tumor cell and macrophage populations does not exist for this

387 particular experiment. Flow cytometry, for example, would not be preferable here because 
388 J774.A1 macrophages are not amenable to trypsin treatment, and thus require manual scraping.

389 In addition, standard flow cytometry would struggle to tease out individual counts of each cell

390 type because the data is collected as a relative count to total number of cells gated. As shown in

391 Figure $7 b$, the quantification of relative counts rather than absolute counts would have led the

392 user to believe that macrophage priming actually promotes tumor cell growth as opposed to

393 inhibiting it (tumor cells represent $18.0 \%$ of the total sample in the unprimed control versus

$39436.1 \%$ of the total sample in the LPS + IFN $\gamma$ primed condition). The optical cell cytometer

395 system we have developed, however, can determine absolute counts of both cell types with

396 single-cell accuracy. With the priming of the J774.A1 macrophages, we were able to not only

397 elucidate the tumoricidal activity of the macrophages, but also the inverse nature of macrophage

398 proliferation and cytotoxic potential.

399 In addition to cell count, the microscopy-based cytometer was able to extract

400 morphological data, such as cell size and mean fluorescence intensity, for every cell. The system

401 is also capable of extracting additional parametric data, such as cell eccentricity, orientation,

402 number of neighbors, first closest object distance, and granularity, extending its capacity beyond

403 simply counting, and into cytometry. The cytometer is also compatible with other fluorescent-

404 based assay stains including annexin $\mathrm{V}$ and/or propidium iodide for assessing cell death, CFSE

405 or BrdU for measuring cell proliferation, and alamarBlue or calcein AM for determining cell

406 viability. For many fields of biology where the interaction between multiple cell types is

407 important, the ability to conduct this type of co-culture experiment may prove invaluable.

408 Suggestions for improving and expanding the capacities of the optical cell cytometer for

409 co-culture as well as mono-culture studies can be found in the supplementary section. They

410 include recommendations for optimizing workflow, handling of an increasing number of 
411 cells/parameters in a single experimental setup (multiplexing), management of multinucleated

412 cells, processing of tissue samples, and analysis of poorly adherent cells. An overview of the

413 technical specifications of the optical cell cytometer can also be found in the supplementary

414 (Additional File 4: Text S1).

415 Conclusions

416

All together, the optical cell cytometer is a viable alternative to flow cytometry, and other

417 currently available imaging cytometers, and is available at only a fraction of the cost for labs that

418 already possess a fluorescent microscope. The system is automated and high throughput using

419 tools already available in most labs. Optical cell counting offers unprecedented sensitivity, going

420 down to the single-cell level, while still boasting an impressive dynamic range limited only by

421 the size of the well plate used. Further, the use of whole-well images allows for quantification

422 across the entire experimental space conferring single-cell accuracy. In addition to cell counts,

423 other features can be gathered from each experiment, including the spatial distribution of cells as

424 well as various morphological analyses. The system is also compatible with other fluorescent-

425 based assay stains. Since the system is put together by the user, it is highly customizable, and

426 allows for direct assessment of assay performance.

427 The microscopy-based cytometer has the added benefit of assessing adherent cells

428 directly on the plate without needing to bring them into solution. As a result, the system does not

429 require caustic trypsin treatment to resuspend cells in solution, as is the case with flow

430 cytometry. One of the most promising aspects of the system is that multiple cell types can be

431 plated together and absolute counts of each population can be elucidated. For many fields of

432 biology, absolute counts can help tease out interesting relationships between cell populations that

433 might not be discernible using relative counts. After imaging and analysis, population statistics- 
434 including cell counts and fluorescence intensity — can be extracted, thresholded, and displayed in

435 histogram or dot-plot form much like the output of a flow cytometer. Overall, we believe the

436 optical cell cytometer will improve the quality of cell cytometric studies and open up the

437 possibility of a new class of experiments centered around the ability to assess multiple cell types

438 in a co-culture setup. The impressive sensitivity and dynamic range of the instrument are also

439 strongly compelling.

440

\section{Acknowledgements}

442 We would like to thank the Miller lab for providing the Nikon Eclipse Ti-E inverted fluorescent

443 microscope and the Drezek lab for editorial assistance.

444

445 Data Availability

446 Raw data files can be found at

447 https://www.dropbox.com/sh/it4e56my94xmlba/AADRuhXlkkX1xnziAu4bYJlLa?dl=0

448

\section{References}

450

451

452

453

454

455

456

457

458

459

460

461

462

463
Arrigoni C, Bersini S, Gilardi M, and Moretti M. 2016. In Vitro Co-Culture Models of Breast Cancer Metastatic Progression towards Bone. Int J Mol Sci 17. 10.3390/ijms17091405

Batista U, Garvas M, Nemec M, Schara M, Veranic P, and Koklic T. 2010. Effects of different detachment procedures on viability, nitroxide reduction kinetics and plasma membrane heterogeneity of V-79 cells. Cell Biol Int 34:663-668. 10.1042/CBI20090276

Bidarra SJ, Barrias CC, Barbosa MA, Soares R, Amedee J, and Granja PL. 2011. Phenotypic and proliferative modulation of human mesenchymal stem cells via crosstalk with endothelial cells. Stem Cell Res 7:186-197. 10.1016/j.scr.2011.05.006

Biswas SK, and Sodhi A. 2002. In vitro activation of murine peritoneal macrophages by monocyte chemoattractant protein-1: upregulation of CD11b, production of proinflammatory cytokines, and the signal transduction pathway. J Interferon Cytokine Res 22:527-538. 10.1089/10799900252982007 
464 Bronner-Fraser M. 1985. Alterations in neural crest migration by a monoclonal antibody

465

466

467

468

469

470

471

472

473

474

475

476

477

478

479

480

481

482

483

484

485

486

487

488

489

490

491

492

493

494

495

496

497

498

499

500

501

502

503

504

505

506

507

508

509 that affects cell adhesion. J Cell Biol 101:610-617.

Bundscherer A, Malsy M, Lange R, Hofmann P, Metterlein T, Graf BM, and Gruber M. 2013. Cell harvesting method influences results of apoptosis analysis by annexin $\mathrm{V}$ staining. Anticancer Res 33:3201-3204.

Carpenter AE, Jones TR, Lamprecht MR, Clarke C, Kang IH, Friman 0, Guertin DA, Chang JH, Lindquist RA, Moffat J, Golland P, and Sabatini DM. 2006. CellProfiler: image analysis software for identifying and quantifying cell phenotypes. Genome Biol 7:R100. 10.1186/gb-2006-7-10-r100

Chakrabarti R, Kundu S, Kumar S, and Chakrabarti R. 2000. Vitamin A as an enzyme that catalyzes the reduction of MTT to formazan by vitamin C. J Cell Biochem 80:133-138.

Chan GK, Kleinheinz TL, Peterson D, and Moffat JG. 2013. A simple high-content cell cycle assay reveals frequent discrepancies between cell number and ATP and MTS proliferation assays. PLoS One 8:e63583. 10.1371/journal.pone.0063583

Chang L, Rissin DM, Fournier DR, Piech T, Patel PP, Wilson DH, and Duffy DC. 2012. Single molecule enzyme-linked immunosorbent assays: theoretical considerations. $J$ Immunol Methods 378:102-115. 10.1016/j.jim.2012.02.011

Cruickshank SM, McVay LD, Baumgart DC, Felsburg PJ, and Carding SR. 2004. Colonic epithelial cell mediated suppression of CD4 T cell activation. Gut 53:678-684.

de Goer de Herve MG, Dembele B, Vallee M, Herr F, Cariou A, and Taoufik Y. 2010. Direct CD4 help provision following interaction of memory CD4 and CD8 T cells with distinct antigen-presenting dendritic cells. J Immunol 185:1028-1036. 10.4049/jimmunol.0904209

Doak SH, Griffiths SM, Manshian B, Singh N, Williams PM, Brown AP, and Jenkins GJ. 2009. Confounding experimental considerations in nanogenotoxicology. Mutagenesis 24:285-293. 10.1093/mutage/gep010

Frgala T, Kalous O, Proffitt RT, and Reynolds CP. 2007. A fluorescence microplate cytotoxicity assay with a 4-log dynamic range that identifies synergistic drug combinations. Mol Cancer Ther 6:886-897. 10.1158/1535-7163.MCT-04-0331

Gedye CA, Hussain A, Paterson J, Smrke A, Saini H, Sirskyj D, Pereira K, Lobo N, Stewart J, Go C, Ho J, Medrano M, Hyatt E, Yuan J, Lauriault S, Meyer M, Kondratyev M, van den Beucken T, Jewett M, Dirks P, Guidos CJ, Danska J, Wang J, Wouters B, Neel B, Rottapel R, and Ailles LE. 2014. Cell surface profiling using high-throughput flow cytometry: a platform for biomarker discovery and analysis of cellular heterogeneity. PLoS One 9:e105602. 10.1371/journal.pone.0105602

Gerashchenko BI. 2008. Quantitative assessment of cell proliferation in the co-culture of mixed cell populations by flow cytometry. Cytometry A 73:492-493. 10.1002/cyto.a.20546

Gerashchenko BI, and Howell RW. 2003. Cell proximity is a prerequisite for the proliferative response of bystander cells co-cultured with cells irradiated with gamma-rays. Cytometry $A$ 56:71-80. 10.1002/cyto.a.10092

Gerashchenko BI, and Howell RW. 2013. Flow cytometry-based quantification of cell proliferation in the mixed cell co-culture. Curr Protoc Cytom Chapter 9:Unit9 40. 10.1002/0471142956.cy0940s63

Goers L, Freemont P, and Polizzi KM. 2014. Co-culture systems and technologies: taking synthetic biology to the next level. J R Soc Interface 11. 10.1098/rsif.2014.0065 
510 Hamid R, Rotshteyn Y, Rabadi L, Parikh R, and Bullock P. 2004. Comparison of alamar blue

511

512

513

514

515

516

517

518

519

520

521

522

523

524

525

526

527

528

529

530

531

532

533

534

535

536

537

538

539

540

541

542

543

544

545

546

547

548

549

550

551

552

553

554

555 and MTT assays for high through-put screening. Toxicol In Vitro 18:703-710. 10.1016/j.tiv.2004.03.012

Hodgkin PD, Lee JH, and Lyons AB. 1996. B cell differentiation and isotype switching is related to division cycle number. J Exp Med 184:277-281.

Holt DJ, Chamberlain LM, and Grainger DW. 2010. Cell-cell signaling in co-cultures of macrophages and fibroblasts. Biomaterials 31:9382-9394. 10.1016/j.biomaterials.2010.07.101

Jones TR, Carpenter A, and Golland P. 2005. Voronoi-Based Segmentation of Cells on Image Manifolds. In: Liu Y, Jiang T, and Zhang C, eds. Computer Vision for Biomedical Image Applications: First International Workshop, CVBIA 2005, Beijing, China, October 21, 2005 Proceedings. Berlin, Heidelberg: Springer Berlin Heidelberg, 535-543.

Katt ME, Placone AL, Wong AD, Xu ZS, and Searson PC. 2016. In Vitro Tumor Models: Advantages, Disadvantages, Variables, and Selecting the Right Platform. Front Bioeng Biotechnol 4:12.10.3389/fbioe.2016.00012

Krtolica A, Ortiz de Solorzano C, Lockett S, and Campisi J. 2002. Quantification of epithelial cells in coculture with fibroblasts by fluorescence image analysis. Cytometry 49:7382. 10.1002/cyto.10149

Lin Y, Trouillon R, Safina G, and Ewing AG. 2011. Chemical analysis of single cells. Anal Chem 83:4369-4392. 10.1021/ac2009838

Lyons AB, and Parish CR. 1994. Determination of lymphocyte division by flow cytometry. $J$ Immunol Methods 171:131-137.

Miki Y, Ono K, Hata S, Suzuki T, Kumamoto H, and Sasano H. 2012. The advantages of coculture over mono cell culture in simulating in vivo environment. J Steroid Biochem Mol Biol 131:68-75. 10.1016/j.jsbmb.2011.12.004

Nasi M, De Biasi S, Bianchini E, Gibellini L, Pinti M, Scacchetti T, Trenti T, Borghi V, Mussini C, and Cossarizza A. 2015. Reliable and accurate CD4+ T cell count and percent by the portable flow cytometer CyFlow MiniPOC and "CD4 Easy Count Kit-Dry", as revealed by the comparison with the gold standard dual platform technology. PLoS One 10:e0116848. 10.1371/journal.pone.0116848

Nose A, and Takeichi M. 1986. A novel cadherin cell adhesion molecule: its expression patterns associated with implantation and organogenesis of mouse embryos. J Cell Biol 103:2649-2658.

Novotney M, Chang ZL, Uchiyama H, and Suzuki T. 1991. Protein kinase C in tumoricidal activation of mouse macrophage cell lines. Biochemistry 30:5597-5604.

Packard BZ, and Komoriya A. 2008. Intracellular protease activation in apoptosis and cellmediated cytotoxicity characterized by cell-permeable fluorogenic protease substrates. Cell Res 18:238-247.10.1038/cr.2008.17

Pozarowski P, Holden E, and Darzynkiewicz Z. 2013. Laser scanning cytometry: principles and applications-an update. Methods Mol Biol 931:187-212. 10.1007/978-1-62703056-4_11

Quent VM, Loessner D, Friis T, Reichert JC, and Hutmacher DW. 2010. Discrepancies between metabolic activity and DNA content as tool to assess cell proliferation in cancer research. J Cell Mol Med 14:1003-1013. 10.1111/j.1582-4934.2010.01013.x Rissin DM, Kan CW, Campbell TG, Howes SC, Fournier DR, Song L, Piech T, Patel PP, Chang L, Rivnak AJ, Ferrell EP, Randall JD, Provuncher GK, Walt DR, and Duffy DC. 2010. 
556

557

558

559

560

561

562

563

564

565

566

567

568

569

570

571

572

573

574

575

576

577

578

579

580

581

582

583
Single-molecule enzyme-linked immunosorbent assay detects serum proteins at subfemtomolar concentrations. Nat Biotechnol 28:595-599. 10.1038/nbt.1641

Schoenborn JR, and Wilson CB. 2007. Regulation of interferon-gamma during innate and adaptive immune responses. Adv Immunol 96:41-101. 10.1016/S00652776(07)96002-2

Spink BC, Cole RW, Katz BH, Gierthy JF, Bradley LM, and Spink DC. 2006. Inhibition of MCF7 breast cancer cell proliferation by MCF-10A breast epithelial cells in coculture. Cell Biol Int 30:227-238. 10.1016/j.cellbi.2005.11.006

Suzuki S, Sato M, Senoo H, and Ishikawa K. 2004. Direct cell-cell interaction enhances proMMP-2 production and activation in co-culture of laryngeal cancer cells and fibroblasts: involvement of EMMPRIN and MT1-MMP. Exp Cell Res 293:259-266.

Ulukaya E, Colakogullari M, and Wood EJ. 2004. Interference by anti-cancer chemotherapeutic agents in the MTT-tumor chemosensitivity assay. Chemotherapy 50:43-50. 10.1159/000077285

Vincent L, and Soille P. 1991. Watersheds in digital spaces: an efficient algorithm based on immersion simulations. IEEE Transactions on Pattern Analysis and Machine Intelligence 13:583-598. 10.1109/34.87344

Vistica DT, Skehan P, Scudiero D, Monks A, Pittman A, and Boyd MR. 1991. Tetrazoliumbased assays for cellular viability: a critical examination of selected parameters affecting formazan production. Cancer Res 51:2515-2520.

Weston SA, and Parish CR. 1990. New fluorescent dyes for lymphocyte migration studies. Analysis by flow cytometry and fluorescence microscopy. J Immunol Methods 133:87-97. 


\section{Figure 1}

Workflow schematic.

Using tools available in most labs, we have created a relatively simple optical counting setup for the quantification of multiple adherent cell types in a multiwell plate. After plating cells, adding condition, and fluorescent staining, the proposed system uses a standard fluorescent microscope to capture whole-well images. Image data is then run through Image for preprocessing and CellProfiler for analysis to generate both absolute cell counts for every well as well as morphological values. Furthermore, utilizing a combination of staining techniques, multiple cell types in a co-culture setup (Cell $A \& B$ as an example) can be uniquely identified and absolutely counted.

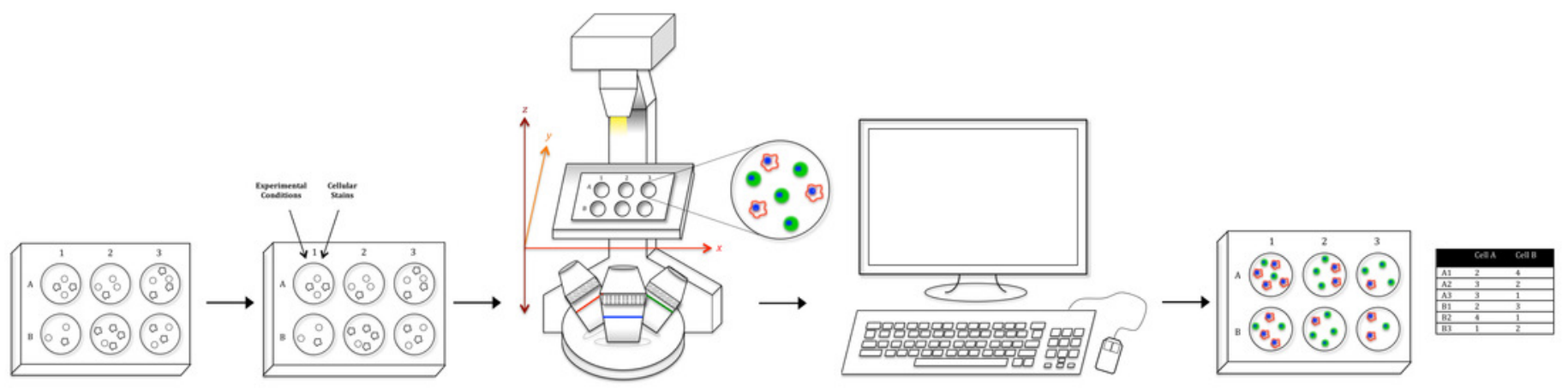




\section{Figure 2}

Automated, whole-well imaging.

Using a fluorescent microscope with motorized $x, y$, and $z$ stage, images spanning the entire well can be sequentially captured and stitched together to produce a high resolution, wholewell image. In the image series above, JC CRL 2116 cell nuclei stained with DAPI were imaged on a 48-well plate with a $10 x$ objective and stitched together. Sequential zooms of the wholewell image demonstrate the incredibly high resolution of the photo, which enables single-cell counting and analysis using a cell segmentation software like CellProfiler (used to generate the final mask).

*Note: Auto Gamma Correction was used for the image. This only affects the reviewing manuscript. See original source image if needed for review.
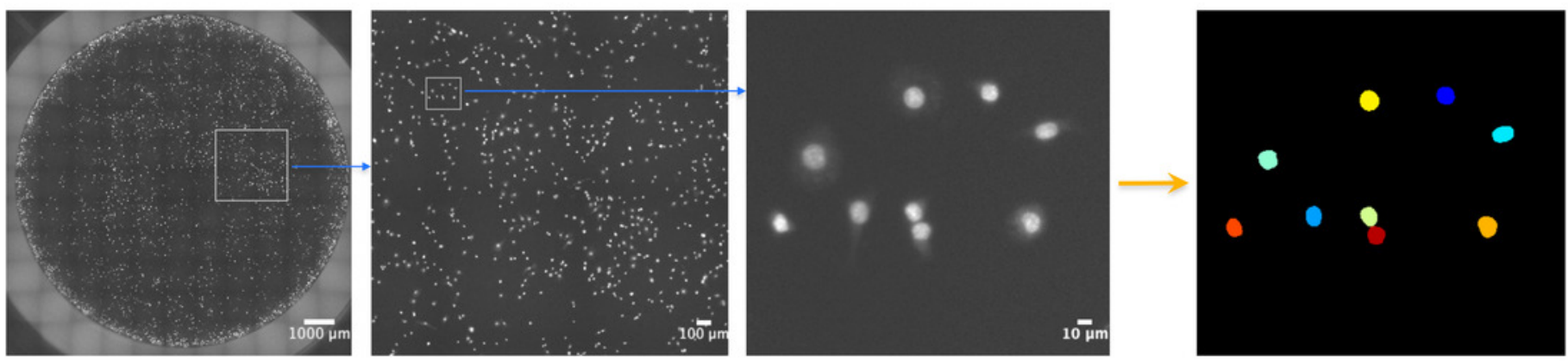


\section{Figure 3}

Power of whole-well imaging and nuclear quantification.

A linear dilution series of JC CRL 2116 cells going from 10,000 cells/well down to 1,000 cells/well was plated on a 48-well plate and given 24 hours to adhere to the surface. Wholewell images of cell nuclei stained with DAPI were then captured and processed to produce either (a) five random images, (b) box crop, or (c) whole-well images, which were then used to determine cell count and generate a linear curve. Accuracy improves significantly as the percent of the experimental space being assayed increases. Error bars represent the standard deviation between triplicate conditions.
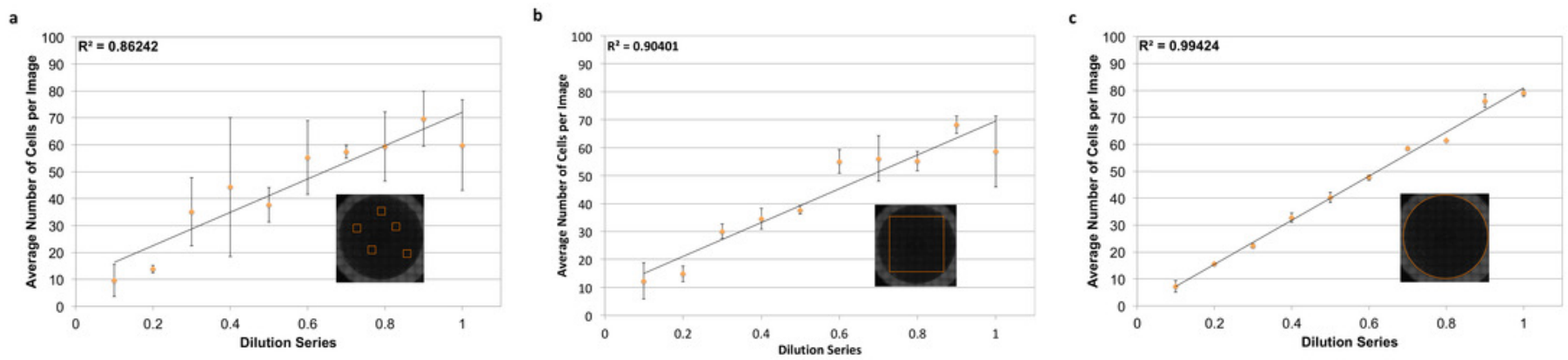


\section{Figure 4}

Determining dynamic range.

A dilution series of JC CRL 2116 cells going from 100,000 cells/well down to 100 cells/well were plated on a 12-well plate (an experiment spanning three orders of magnitude) and given 24 hours to adhere to the surface. Cell nuclei stained with DAPI were then quantified and used to generate a linear curve. The system performs robustly over a wide dynamic range and is limited only by the surface area of the multiwell. Error bars represent the standard deviation between triplicate conditions.

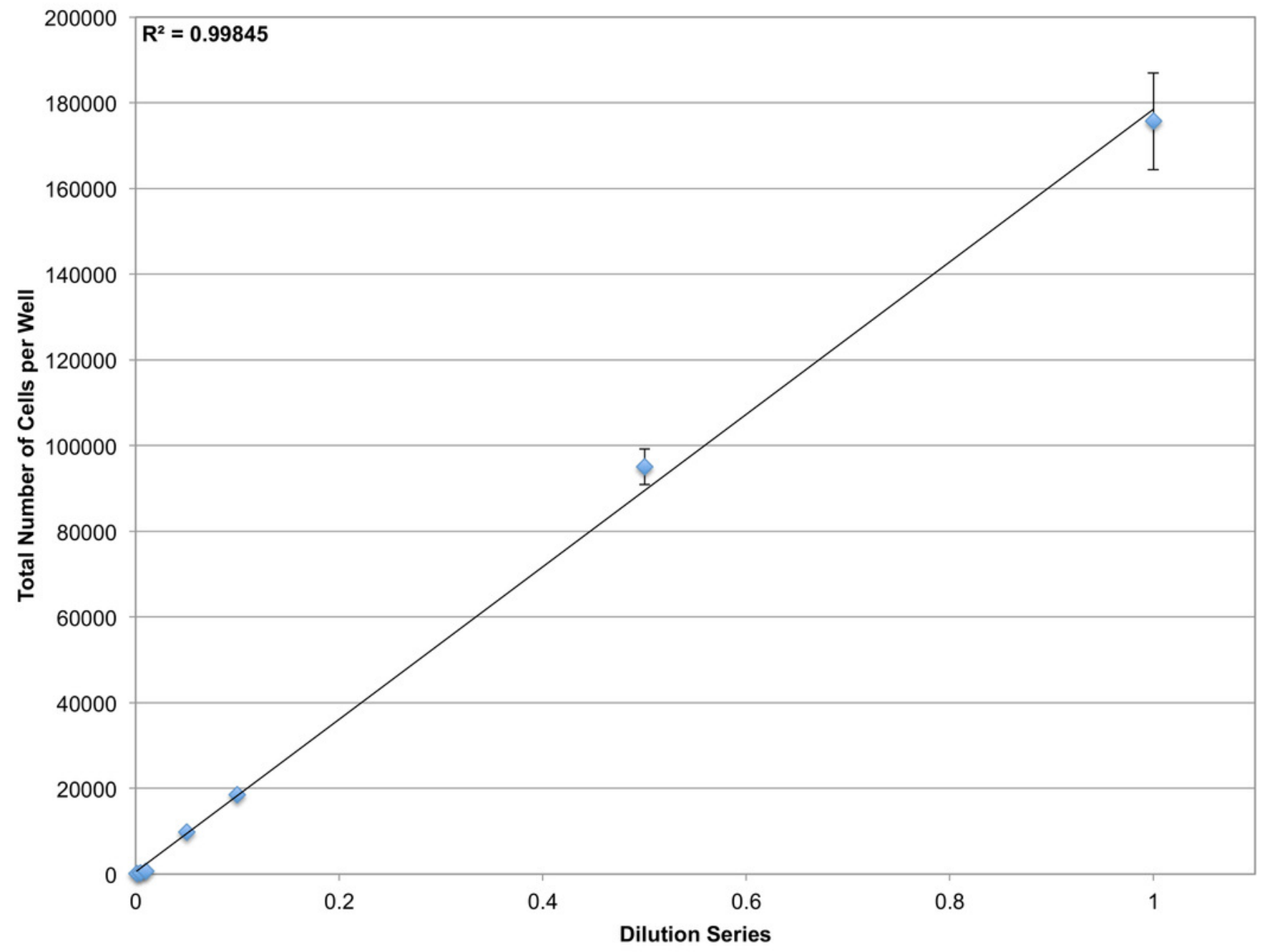


Figure 5

Establishing sensitivity.

Various concentrations of JC CRL 2116 cells going from 100 cells/well down to 1 cell/well were plated on a 48-well plate and given 24 hours to adhere to the surface. CellProfiler-derived counts of DAPI stained nuclei were compared with cell counts obtained by human assessment of associated brightfield images for each well. The system performs robustly even at the single-cell level $\left(R^{2}=0.98\right.$, slope $\left.=0.86\right)$ with most deviations attributable to human error when manually counting.

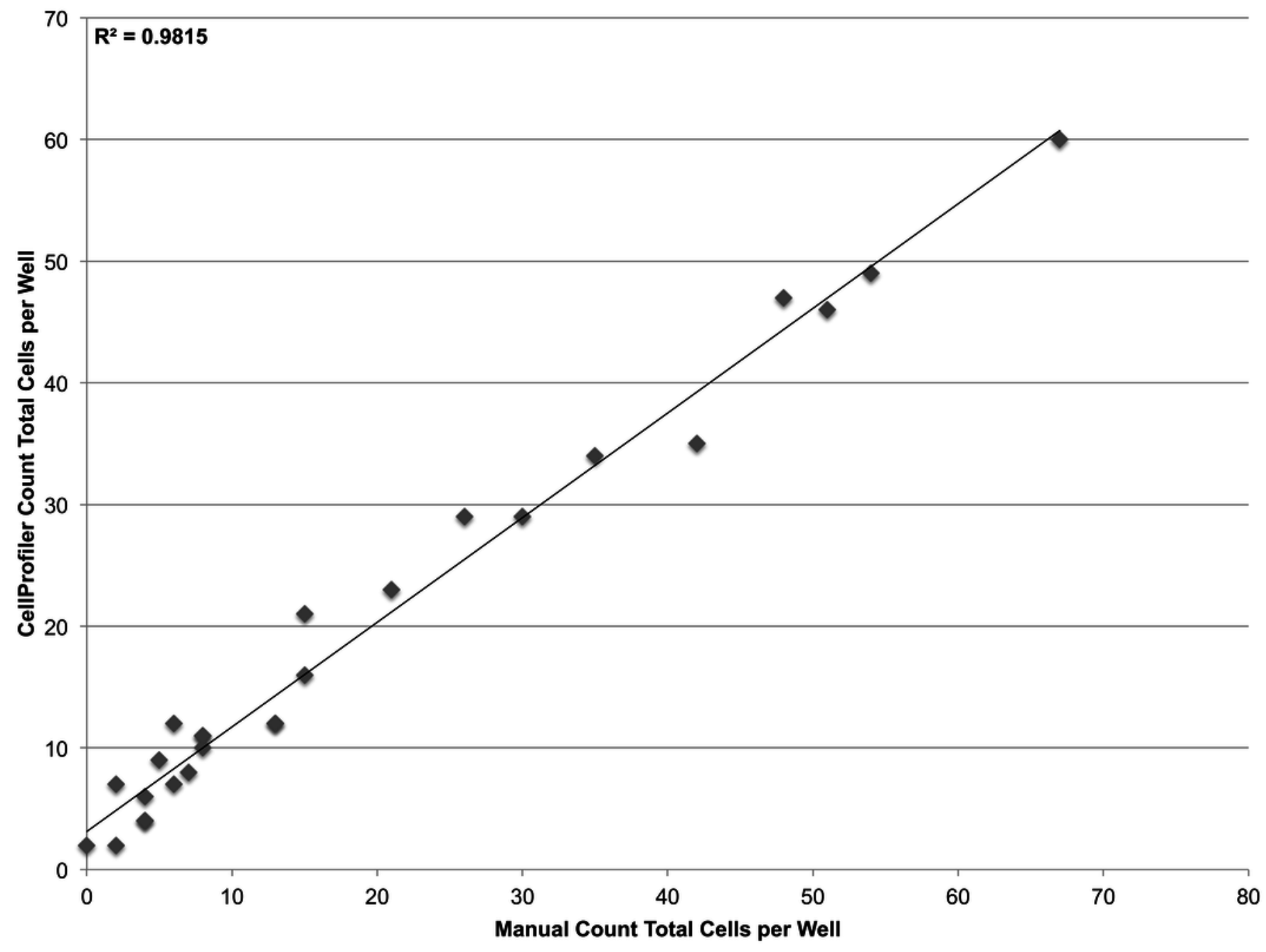




\section{Figure 6}

Secondary quantification of surface and cytoplasmic stains using nuclei as seeds.

A linear dilution series of 7774 .A1 cells going from 10,000 cells/well down to 1,000 cells/well was plated on a 48-well plate and given 24 hours to adhere to the surface. Cells were stained with Vybrant CFDA SE (cytoplasmic stain), phycoerythrin (PE)-conjugated anti-CD11b antibodies (surface stain), and DAPI (nuclear stain). Using nuclei as seeds, cells delineated by a surface or cytoplasmic stain could be accurately quantified as demonstrated by the nearperfect $\mathrm{R}^{2}$. Error bars represent the standard deviation between triplicate conditions.

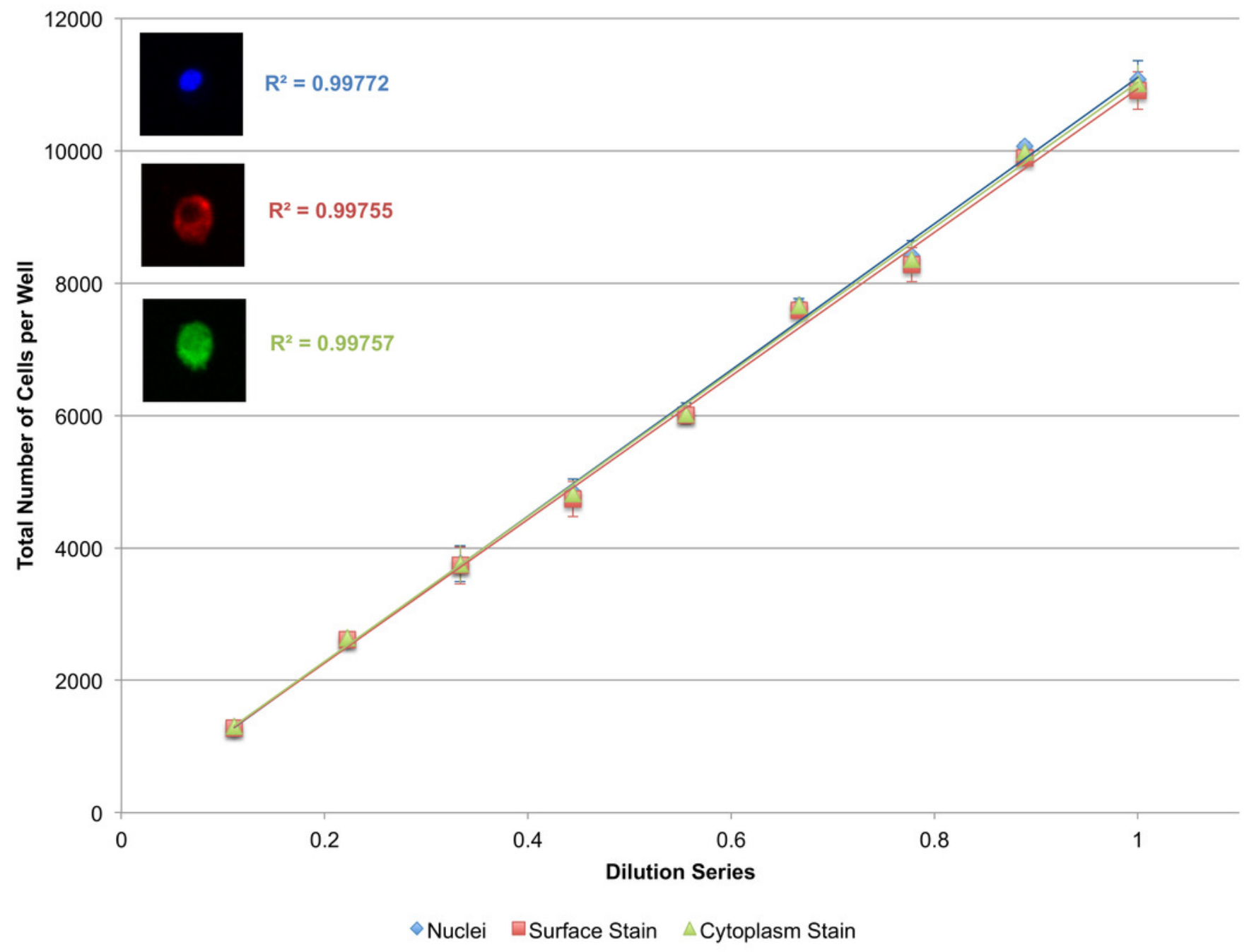




\section{Figure 7}

Tumoricidal properties of primed macrophages in the presence of co-cultured tumor cells.

J774.A1 macrophages were primed with either IFNY, LPS, or both and subsequently cocultured with JC CRL 2116 tumor cells. J774.A1 cells were labeled with PE-conjugated antiCD11b antibodies (red surface stain) while JC CRL 2116 cells were labeled with Vybrant (green cytoplasmic stain). Both cells were also stained with DAPI. (a) Normalized and (b) absolute cell counts as well as (c) cell area were determined for each condition. (d) CD11b expression levels as well as macrophage uptake/phagocytic activity were calculated by extracting the mean fluorescent intensity of macrophage PE and Vybrant staining respectively. The experiment reveals an interesting, dynamic relationship between activated macrophages and target tumor cells that would have been missed by any setup that looks at relative counts. Data for (a), (c), and (d) are normalized to the unprimed control. Error bars represent the standard deviation between triplicate conditions. 
a

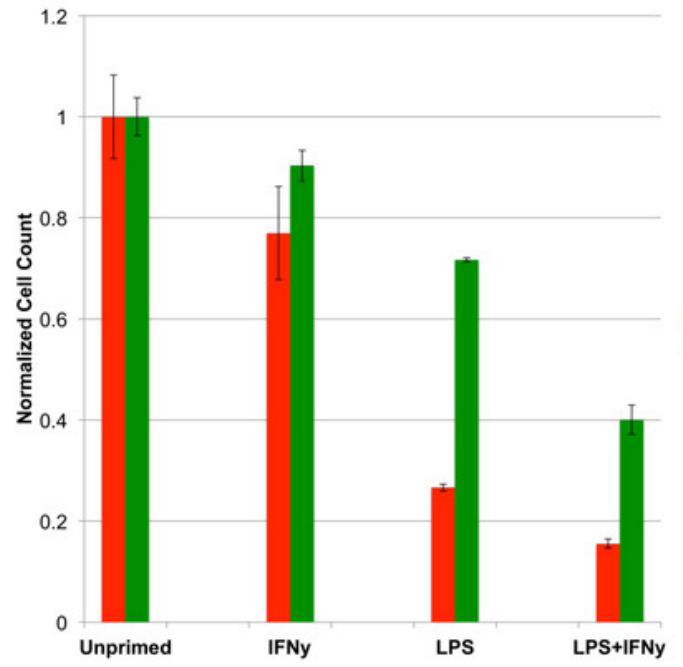

c

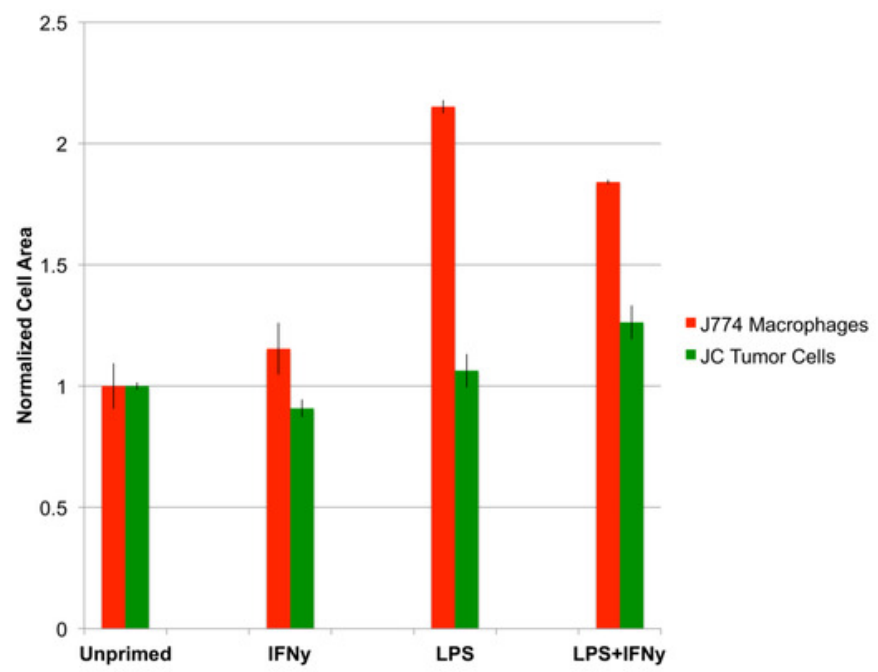

b

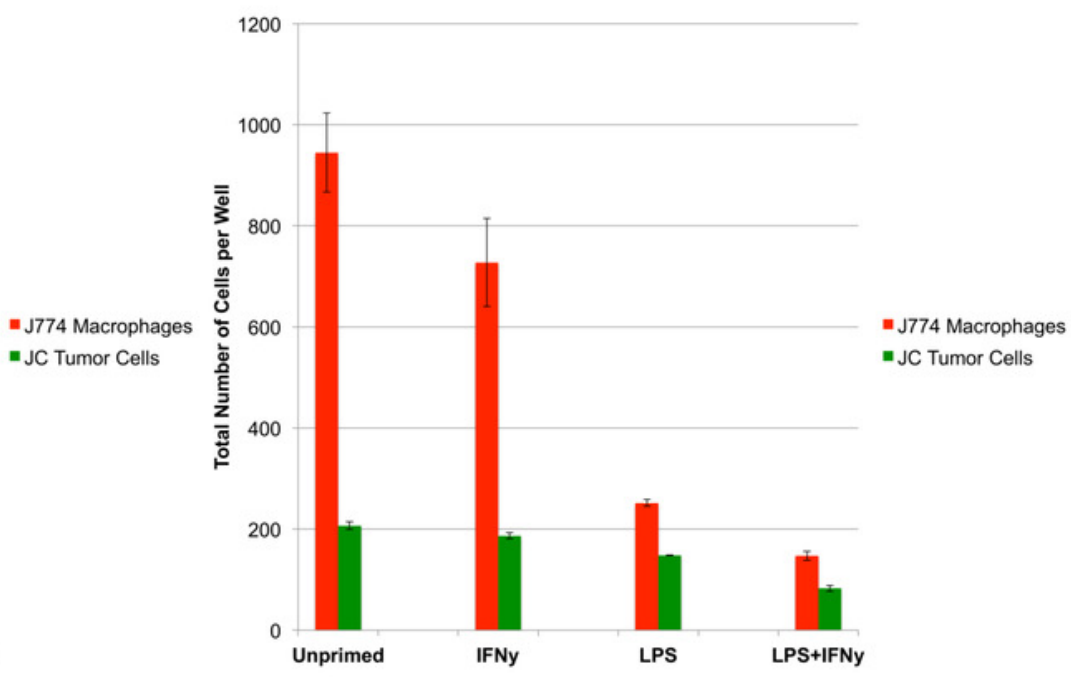

d

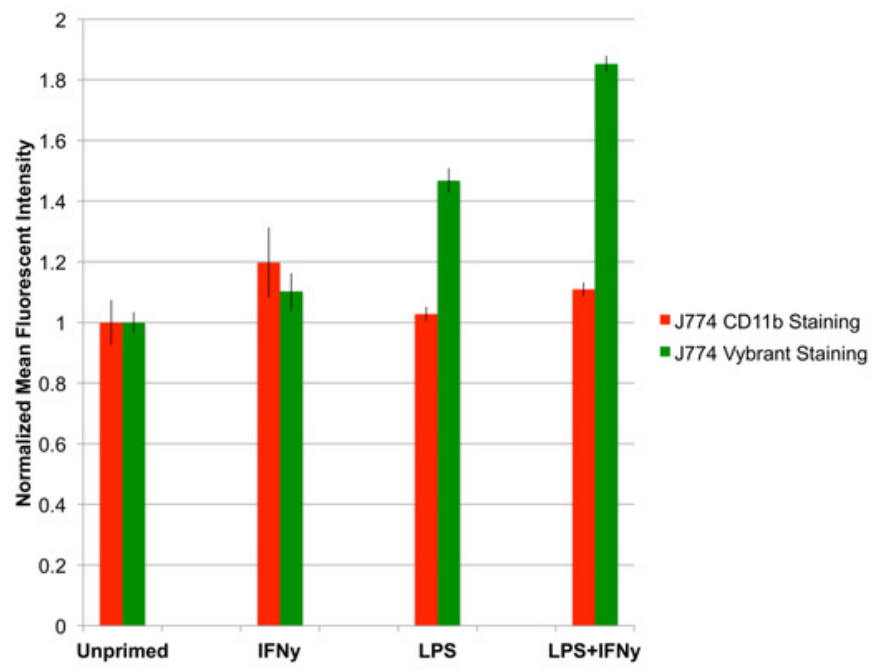

